\title{
Transcriptional repression of CDKN2D by PML/RARa contributes to the altered proliferation and differentiation block of acute promyelocytic leukemia cells
}

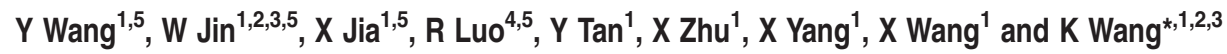

Cell proliferation and differentiation are highly coordinated processes. These two processes are disrupted during leukemogenesis, resulting in differentiation block and uncontrolled proliferation in leukemia. To understand the mechanisms disrupting the coordination between the two processes in acute promyelocytic leukemia (APL), we investigated the regulatory mechanism of the negative cell cycle regulator CDKN2D by the promyelocytic leukemia/retinoic acid receptor $\alpha$ (PML/RAR $\alpha$ ) fusion protein and the role of CDKN2D in cell differentiation and proliferation. We found that CDKN2D expression in APL cells was significantly lower than that in normal promyelocytes. By chromatin immunoprecipitation and luciferase reporter assays, we showed that PML/RAR $\alpha$ directly bound to and inhibited the transactivation of the CDKN2D promoter. Further evidence by the truncated and mutated CDKN2D promoters revealed that the everted repeat 8 (ER8) motif on the promoter was the binding site of PML/RAR $\alpha$. Forced expression of CDKN2D induced G0/G1 phase arrest and partial granulocytic differentiation in APL-derived NB4 cells, suggesting the function of CDKN2D in regulating both cell proliferation and granulocytic differentiation. Furthermore, all-trans retinoic acid (ATRA) significantly induced CDKN2D expression in APL cells and knockdown of CDKN2D expression during ATRA treatment partially blocked the ATRA-induced differentiation and cell cycle arrest. Collectively, our data indicate that CDKN2D repression by PML/RAR $\alpha$ disrupts both cell proliferation and differentiation in the pathogenesis of APL, and induced expression of CDKN2D by ATRA alleviates the disruption of both processes to ensure treatment efficiency. This study provides a mechanism for coupling proliferation and differentiation in leukemic cells through the action of CDKN2D.

Cell Death and Disease (2014) 5, e1431; doi:10.1038/cddis.2014.388; published online 2 October 2014

Acute promyelocytic leukemia (APL), a distinct subtype of acute myeloid leukemia, is characterized by an oncogenic fusion protein of a translocation between chromosomes 15 and 17, promyelocytic leukemia/retinoic acid receptor $a(\mathrm{PML} /$ RARa). PML/RARa has an essential role in the leukemogenesis of APL by interfering with its target genes, eventually leading to a differentiation block at the promyelocytic stage and a hyperproliferation of blocked promyelocytes; ${ }^{1}$ both are the hallmark features of APL. Pharmacological concentrations of all-trans retinoic acid (ATRA) can induce the degradation of PML/RAR $a$ and restore the expression of those genes suppressed by PML/RARa, resulting in the granulocytic differentiation and cell cycle arrest of leukemic cells. ${ }^{2}$

It has been well documented that cell proliferation and differentiation are two fundamental and distinct processes that must be coordinated during development and homeostasis. ${ }^{3}$ Loss of coordination between the two processes usually leads to aberrant development and tumor formation. ${ }^{4} \mathrm{~A}$ large number of studies have shown that proliferation and differentiation in hematopoietic cells are modulated by different regulators. Cell proliferation is mainly controlled by the cell cycle machinery and apoptosis; ${ }^{5}$ myeloid differentiation is generally orchestrated by tightly tuned activities of hematopoietic transcription factors. ${ }^{6}$ Accordingly, during the pathogenesis of APL, abnormal differentiation block and uncontrolled cell proliferation are, respectively, attributed to disruption of differentiation- and proliferation-related regulators by PML/RAR $a$. For example, PML/RARa blocks differentiation by interfering with the normal function of RAR $a$ and PU.1. ${ }^{7,8}$ On the other hand, PML/RAR $a$ alters cell proliferation by impairing the formation of functional PML nuclear bodies, which suppresses cell growth by inducing G1-phase arrest and apoptosis. ${ }^{9,10}$

Interestingly, emerging evidence suggests that cell differentiation and proliferation can be controlled by the same regulators. Certain dual-function regulators may serve as links

\footnotetext{
${ }^{1}$ State Key Laboratory of Medical Genomics and Shanghai Institute of Hematology, Rui Jin Hospital, Shanghai Jiao Tong University School of Medicine, Shanghai, 200025, China; ${ }^{2}$ Institute of Health Sciences, Shanghai Institutes for Biological Sciences, Chinese Academy of Sciences, Shanghai, 200025, China; ${ }^{3}$ Sino-French Research Center for Life Sciences and Genomics, Rui Jin Hospital, Shanghai Jiao Tong University School of Medicine, Shanghai, 200025, China and ${ }^{4}$ School of Life Sciences/Center for Computational Systems Biology, Fudan University, Shanghai, 200433, China

${ }^{*}$ Corresponding author: K Wang, State Key Laboratory of Medical Genomics and Shanghai Institute of Hematology, Rui Jin Hospital, Shanghai Jiao Tong University School of Medicine, 197 Ruijin Er Rd, Shanghai, 200025, China. Tel: +86 21 64370045; Fax: +86 21 64743206; E-mail: kankanwang @ shsmu.edu.cn

${ }^{5}$ These authors contributed equally to this work.

Abbreviations: APL, acute promyelocytic leukemia; PML/RAR $\alpha$, promyelocytic leukemia/retinoic acid receptor $\alpha$; ATRA, all-trans retinoic acid; RA, retinoic acid; 1,25VD3, 1,25-dihydroxy vitamin D3; RARE, retinoic acid response element

Received 27.1.14; revised 09.7.14; accepted 14.7.14; Edited by Z-X Xiao
} 
for coordinating cell proliferation and differentiation. For example, HoxA10 can simultaneously affect both cell proliferation and differentiation during the development of hematopoiesis, ${ }^{11}$ resulting in precise and highly coordinated developmental changes of blood cells. Dysregulation of HoxA10 participates in perturbing both cell proliferation and differentiation, resulting in the occurrence of acute myeloid leukemia. ${ }^{12}$ However, the molecular mechanisms underlying the coordination of cell proliferation and differentiation are just beginning to be understood. Emerging evidence suggests that cell cycle regulators, especially CDK inhibitors (CKIs), are involved in the regulation of differentiation in addition to their well-documented function of governing the cell cycle process. ${ }^{3}$ On the basis of the exploration of verified PML/ RAR $a$ binding sites derived from several genome-wide screening, 8,13 of particular interest is CDKN2D, one of the INK4 family CKIs encoding p19INK4D.

CDKN2D is a member of the INK4 family CKIs. The INK4 family consists of CDKN2A, CDKN2B, CDKN2C and CDKN2D, and generally regulates the G1-to-S phase transition by specifically inhibiting the activity of CDK4 and CDK6. ${ }^{14}$ The genes of the INK4 family act as tumor suppressors in the pathogenesis of many malignancies, including leukemia, ${ }^{14}$ and provide a direct link between tumorigenesis and the loss of negative control of cell cycle progression. However, the role of the INK4 family participating in the differentiation block of malignant cells has not been clearly explored. It has been reported that, in normal neural development, CDKN2D is capable of deciding whether differentiation starts before or after cell cycle exit, ${ }^{15}$ implicating its potential role in differentiation. In myeloid differentiation, CDKN2D promotes the differentiation and maturation of megakaryocytes by arresting endomitosis, ${ }^{16}$ suggesting the ability of CDKN2D to regulate differentiation. However, it is unclear whether or not CDKN2D is involved in the coordination of abnormal proliferation and differentiation of leukemia cells and the manner by which CDKN2D regulates these processes.

It is worth noting that CDKN2D expression is induced by 1,25-dihydroxy vitamin D3 $(1,25-V D 3)$ or retinoic acid (RA) in multiple cell lines, such as U937 myeloid leukemia cells, SCC25 head and neck squamous cell carcinoma cells and Calu-3 lung carcinoma cells, ${ }^{17}$ suggesting that CDKN2D is a target of nuclear receptors and may be directly regulated by the oncogenic PML/RARa fusion protein. To fully understand the leukemogenesis of APL, it is crucial to determine the role of $\mathrm{PML} / \mathrm{RAR} a$ in the regulation of its target genes that regulate both cell proliferation and differentiation. In this study, we show that $C D K N 2 D$ expression is directly repressed by the PML/ RAR $a$ fusion protein, and the decrease in CDKN2D expression contributes to the altered proliferation and differentiation block of APL cells. ATRA significantly induces CDKN2D expression, and increased expression of $C D K N 2 D$ is linked to ATRA-induced differentiation and cell cycle arrest. Our data suggest the dual nature of CDKN2D in controlling both differentiation and proliferation.

\section{Results}

CDKN2D expression is significantly lower in APL cells than that in normal promyelocytes. We initially compared CDKN2D expression in primary APL patient samples with that in normal promyelocytes. The expression profiles of samples from 14 APL patients, 5 normal promyelocytes and 5 peripheral mononuclear cells ${ }^{18}$ were retrieved to perform the comparison. As shown in Figure 1a, the expression level of CDKN2D in APL patient samples was significantly lower than that in normal promyelocytes and peripheral mononuclear cells. To determine whether CDKN2D expression is inversely correlated with the PML/RARa fusion protein in APL, we performed quantitative real-time RT-polymerase chain reaction (qRT-PCR) analysis to compare CDKN2D expression in a

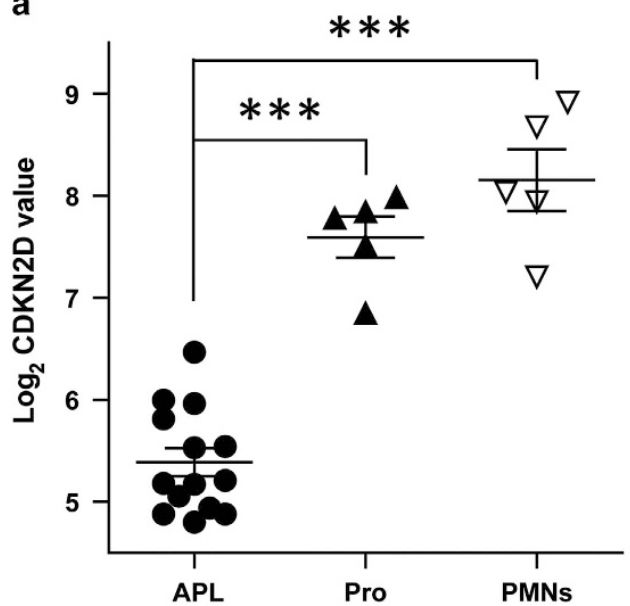

b

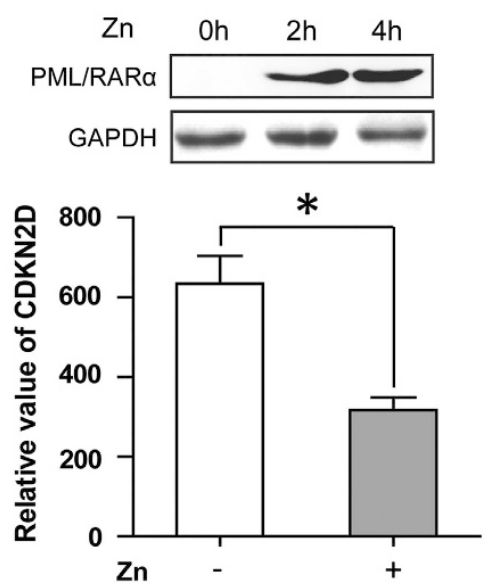

Figure 1 CDKN2D expression is significantly lower in APL cells than that in normal promyelocytes. (a) The expression level of CDKN2D in APL patient samples was significantly lower than that in normal promyelocytes (Pro) and peripheral mononuclear cells (PMNs). The expression data were retrieved from microarray profiling performed by Payton JE et al. (GSE12662). ${ }^{18}$ The expression values are absolute intensities after being log-transformed. (b) The upper panel shows the induced expression of PML/RAR $\alpha$ by $\mathrm{ZnSO}_{4}(\mathrm{Zn})$ in PR9 cells using western blot analysis. The lower panel shows the reduced expression of CDKN2D after the induction of PML/RAR $\alpha$. PR9 cells were treated with or without $100 \mu \mathrm{M} \mathrm{ZnSO}_{4}(\mathrm{Zn})$ for $4 \mathrm{~h}$. The relative mRNA level of CDKN2D was normalized to GAPDH. * indicates $P<0.05,{ }^{* * *}$ indicates $P<0.001$ 
the presence or absence of PML/RARa in PR9 cells, a PML/ RAR $a$-inducible cell line. As shown in Figure $1 \mathrm{~b}, C D K N 2 D$ expression was markedly decreased after the PML/RARa induction. These results suggest that CDKN2D expression can be repressed by PML/RARa in APL cells.

PML/RARa represses the transcription of CDKN2D through the everted repeat 8 (ER8) site on the CDKN2D promoter. To determine whether reduced CDKN2D expression in APL cells is directly mediated by PML/RAR $a$, we cloned a 900-bp fragment of the CDKN2D promoter upstream of the transcriptional start site into a luciferase reporter plasmid to conduct promoter-reporter assays (Figure 2a). Increasing amounts of the pSG5-PML/RARa expression construct were co-transfected into 293T cells along with the CDKN2D promoter plasmid. As shown in Figure 2b, CDKN2D promoter activity was repressed by PML/ RAR $a$ in a dose-dependent manner, indicating that CDKN2D expression was transcriptionally repressed by PML/RAR $a$. To locate the region within the CDKN2D promoter responsible for the PML/RAR $a$-mediated transcriptional repression, a series of $5^{\prime}$ truncated luciferase constructs were cotransfected into 293T cells with the pSG5-PML/RARa expression plasmid. As shown in Figure 2c, promoter activity of the -488 bp truncated form showed no significant change of the repression fold by PML/RARa, as compared with the $-900 \mathrm{bp}$ form, indicating that the region from $-900 \mathrm{bp}$ to
- 488 bp was less sensitive to PML/RARa-mediated inhibitory effect than other regions. In contrast, truncation to -273 bp caused a significant reduction in the degree of PML/ RAR $a$-mediated suppression, suggesting that the region between $-488 \mathrm{bp}$ and $-273 \mathrm{bp}$ might be essential for transcriptional repression of the CDKN2D promoter by PML/RARa.

Analysis of the region between $-488 \mathrm{bp}$ and $-273 \mathrm{bp}$ of the CDKN2D promoter identified a $20 \mathrm{bp}$ sequence (-368 bp to - $349 \mathrm{bp}$ ) organized as an ER8 site, which is an everted repeat of two RA response element (RARE) half sites separated by eight nucleotides. This ER8 site has been reported to be involved in 1,25-VD3- or RA-induced CDKN2D expression in other cell lines, ${ }^{17}$ providing the possibility that this site may be responsible for the PML/RAR $a$-mediated transrepression of CDKN2D in APL. To test this hypothesis, we mutated the ER8 site in the -488-bp construct and found that the repression was significantly relieved when the ER8 site was mutated (Figure 2c). The results obtained in 293T cells could also be seen in APL patient-derived NB4 cells that express the endogenous PML/RARa fusion protein (Figure 2d). Collectively, the mutation analysis demonstrated that PML/RARa repressed the transactivation of the CDKN2D promoter through the ER8 site located at $-368 \mathrm{bp}$ to $-349 \mathrm{bp}$.

PML/RAR $a$ binds to the endogenous CDKN2D promoter. One important question that remains to be answered is a

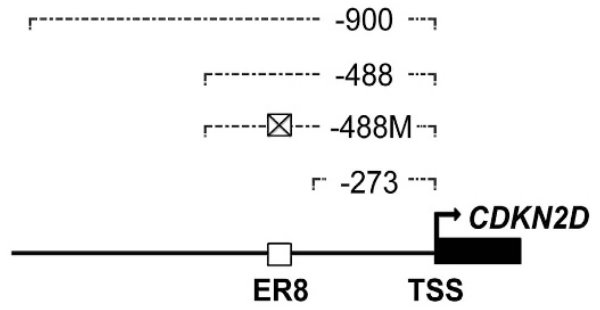

b

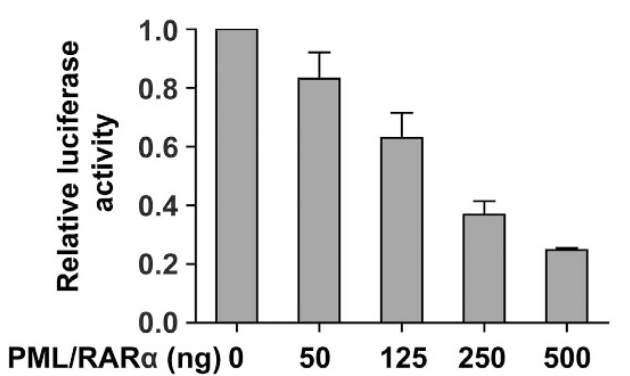

C

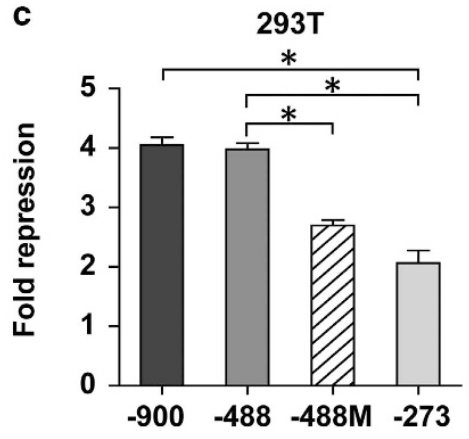

d

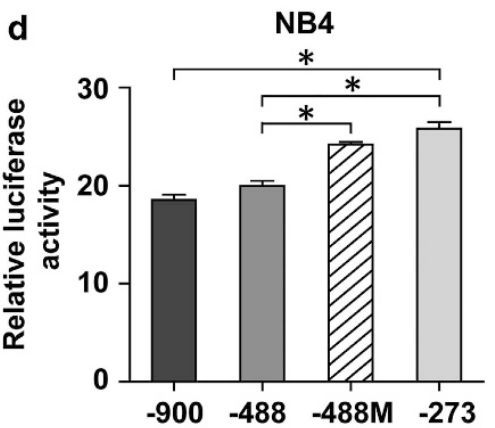

Figure 2 PML/RAR $\alpha$ represses the promoter activity of CDKN2D through the ER8 site. (a) Schematic representation of the CDKN2D promoter region shows the different truncations/mutations used in this study. Numbering is indicated with respect to the transcriptional start site (TSS) of CDKN2D (bent arrow). $\square$ represents the wild-type ER8 site and $\bigotimes$ represents the mutated ER8 site. (b) PML/RAR $\alpha$ repressed the promoter activity of CDKN2D in a dose-dependent manner. The CDKN2D promoter reporter construct (-900 bp) and increasing amounts of PSG5-PML/RAR $\alpha$ expression plasmid (PML/RAR $\alpha$ ) were cotransfected into 293T cells. Luciferase values were normalized to those of control. Values were the mean \pm S.E.M. obtained from at least three independent experiments. (c-d) The ER8 site located between $-273 \mathrm{bp}$ and $-488 \mathrm{bp}$ of the CDKN2D promoter is critical for PML/RAR $\alpha$-mediated repression. The 293T cells were transfected with different truncations/mutations of the CDKN2D promoter reporter construct and the pSG5-PML/RAR $\alpha$ expression plasmid or the empty vector. Results in 293T cells (c) are represented as fold repression. The NB4 cells were transfected with different truncations/ mutations of the CDKN2D promoter reporter construct. Results in NB4 cells (d) are relative to the empty vector (pGL3-basic). ${ }^{*}$ indicates $P<0.05$ 
whether PML/RAR $a$ binds to the CDKN2D promoter in vivo. To address this question, we performed chromatin immunoprecipitation (ChIP)-PCR assays using anti-PML, anti-RARa and a nonspecific antibody (normal $\operatorname{lgG}$ ). Primers were designed to amplify a 207-bp amplicon covering the ER8 site (region 1) (Figure 3a). An irrelevant region (region 2), which was located far upstream of the transcriptional start site and contained no RARE sites, was selected as a negative control. As illustrated in Figure 3b (left panel), we found that the positive band was amplified in the chromatin region covering the ER8 site using the anti-RARa immunoprecipitation in U937 cells, in accordance with a previous study that RARa binds to the ER8 site of the CDKN2D promoter. ${ }^{17}$ Further, we treated PR9 cells with $\mathrm{ZnSO}_{4}$ to induce the expression of PML/RAR $a$ and found that region 1 was highly enriched by both the anti-PML and anti-RARa antibodies, but were not enriched by the nonspecific normal IgG control. In contrast, region 2 was not enriched by all of the antibodies (Figure $3 \mathrm{~b}$, middle panel). These results indicate that when cells express PML/RARa protein, PML/ RAR $a$ could compete with RARa to bind the ER8 site of the $C D K N 2 D$ promoter. To further support the above finding, we performed ChIP assays in the APL-derived NB4 cells. The result was compliant with that in $\mathrm{ZnSO}_{4}$-treated PR9 cells (Figure 3b, right panel). Collectively, the results of ChIP assays show that PML/RARa binds to the endogenous CDKN2D promoter.

Forced expression of CDKN2D induces cell cycle arrest and a partial granulocytic differentiation of APL cells. The findings that $C D K N 2 D$ was targeted and repressed by PML/RARa prompted us to investigate the biological effects of CDKN2D restoration on APL-derived NB4 cells. We restored CDKN2D expression in NB4 cells with retroviral transduction (Figure 4a), and compared the differentiation potential and cell cycle status of cells before and after restoration. As shown in Figure $4 \mathrm{~b}$, forced expression of CDKN2D significantly increased the cells in the G0/G1 phase from $45.3 \%$ to $76.3 \%$ which was in accordance with the welldocumented role of CDKN2D in cell cycle control. Forced expression of CDKN2D also induced a partial granulocytic differentiation of NB4 cells, based on the observations of the elevation of the neutrophilic differentiation marker CD11b (Figure 4c) and the morphological change (Figure 4d). These data suggest that the cell cycle regulator CDKN2D is involved in both cell cycle regulation and granulocytic differentiation.

ATRA restores the expression of CDKN2D in APL cells. APL is characterized by a unique clinical response to ATRA, which is able to efficiently induce differentiation of APL cells and restore the expression of genes inhibited by PML/RAR $a^{2}{ }^{2}$ We thus examined CDKN2D expression in NB4 cells treated with $1 \mu \mathrm{M}$ ATRA in a time course analysis by qRT-PCR. We found that ATRA treatment increased the mRNA level of $C D K N 2 D$ in a time-dependent manner (Figure 5a). Because we found that PML/RARa repressed CDKN2D transcription through the ER8 site, which may serve as a non-canonical site of RA signaling, we further tested whether CDKN2D is a direct ATRA-responsive gene independent of de novo protein synthesis. NB4 cells were pre-treated with or without cycloheximide, a protein synthesis inhibitor, before the addition of ATRA. The results showed that CDKN2D expression induced by ATRA was not perturbed by pretreating NB4 cells with cycloheximide (Figure 5b), indicating that $C D K N 2 D$ was a direct ATRA-responsive gene independent of de novo protein synthesis.

Because the endogenous VDR and RARa can regulate $C D K N 2 D$ expression at the presence of ATRA, we compared CDKN2D expression after ATRA treatment in cells with or without PML/RARa. We treated PR9 cells before and after the addition of $\mathrm{ZnSO}_{4}$ with $1 \mu \mathrm{M}$ ATRA and performed qRT-PCR. As expected, we found that CDKN2D expression was both upregulated after ATRA treatment no matter whether PR9 cells were treated with or without $\mathrm{ZnSO}_{4}$. More interestingly, ATRA treatment induced a stronger (10.5-fold) upregulation of CDKN2D in the presence of PML/RARa, as compared with endogenous RARa (2.9-fold) (Figure 5c), indicating that ATRA induced CDKN2D expression more efficiently in PML/RARa expressing cells.

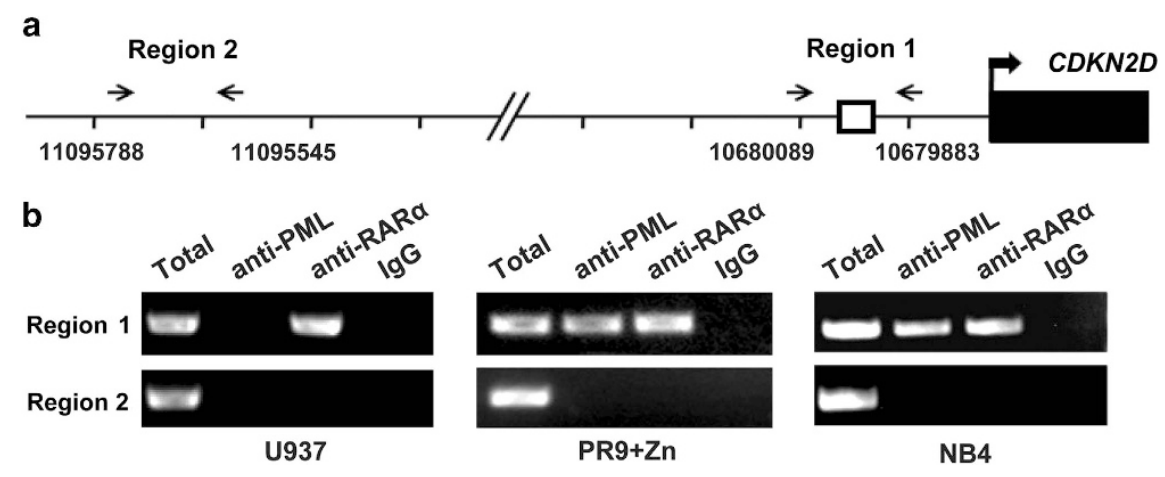

Figure 3 PML/RAR $\alpha$ binds to the endogenous CDKN2D promoter. (a) Schematic representation of the CDKN2D promoter region. The bent arrow represents the transcriptional start site. $\square$ represents the ER8 site. The paired arrowheads represent the primers used in the ChIP analysis. (b) The ChIP assays of PML/RAR $\alpha$ binding within the CDKN2D promoter were performed in U937 cells, $\mathrm{ZnSO}_{4}$-treated PR9 cells and NB4 cells. The total input and immunoprecipitated DNAs were analyzed by PCR using primers specific (region 1) or non-specific (region 2) to the ER8 site. Left: the positive band was amplified in the region 1 using the anti-RAR $\alpha$ immunoprecipitation in U937 cells. Middle: region 1 was highly enriched by both the anti-PML and anti-RAR $\alpha$ antibodies in $\mathrm{ZnSO}_{4}$-treated PR9 cells. PR9 cells were treated with $100 \mu \mathrm{M} \mathrm{ZnSO}{ }_{4}$ for $4 \mathrm{~h}$. Right: region 1 was highly enriched by both the anti-PML and anti-RAR $\alpha$ antibodies in NB4 cells. Total input and DNAs immunoprecipitated by normal lgG served as positive and negative controls, respectively 
b
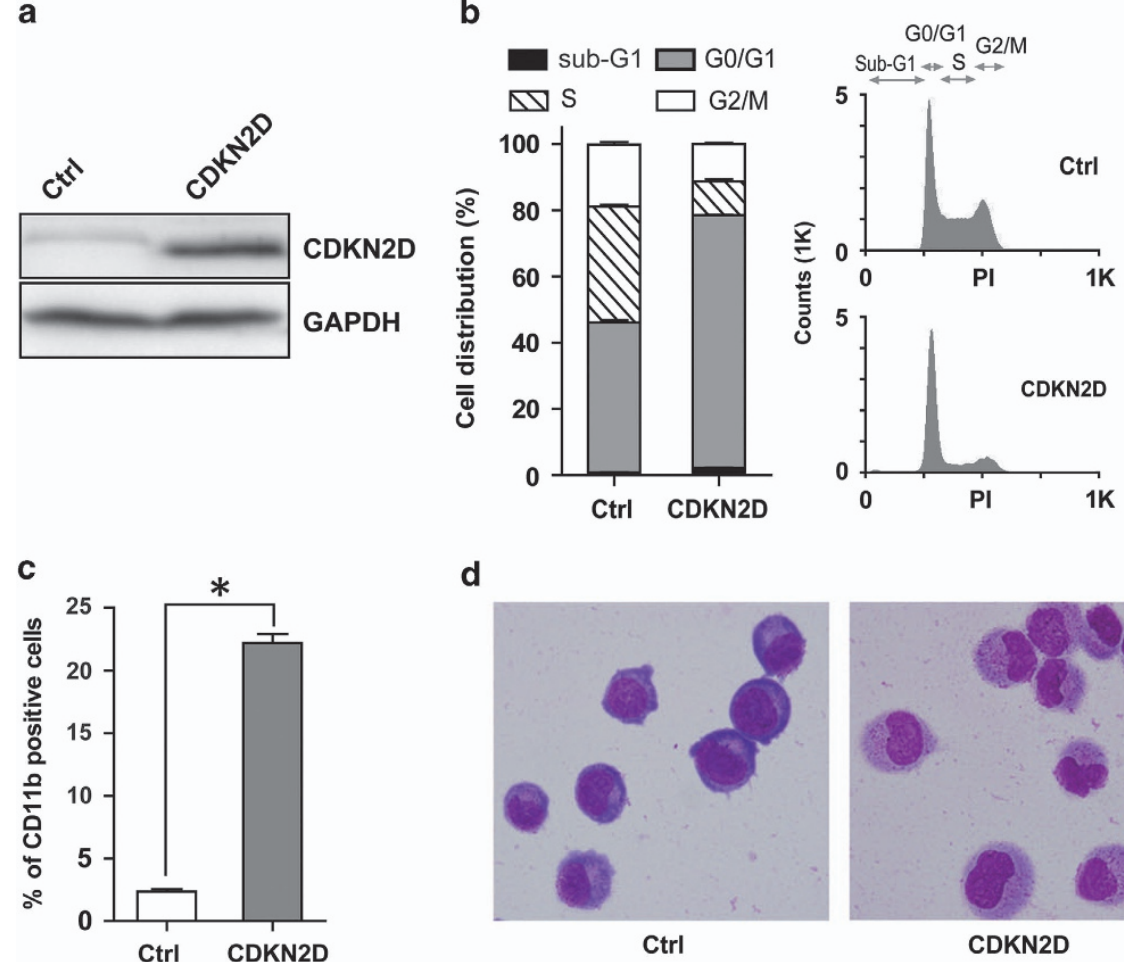

d

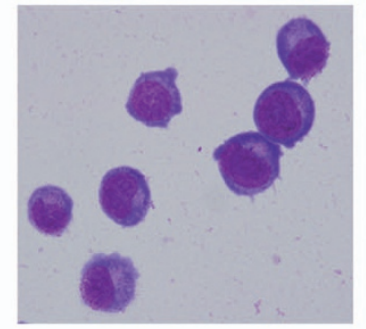

Ctrl

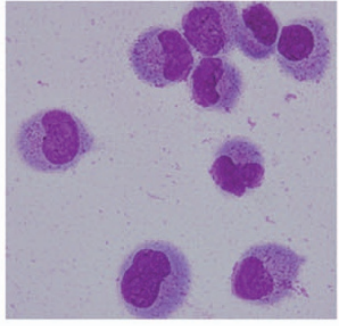

CDKN2D

Figure 4 Ectopic expression of CDKN2D induces cell cycle arrest and a partial granulocytic differentiation of NB4 cells. (a) Western blot analysis of CDKN2D expression in total cell lysates. NB4 cells were harvested by $72 \mathrm{~h}$ after MigR1 (Ctrl) or MigR1-CDKN2D (CDKN2D) infection. (b) Overexpression of CDKN2D induced G0/G1 phase arrest of NB4 cells. Cell cycle distribution was analyzed in GFP-positive cells. Values were the mean \pm S.E.M. obtained from three independent experiments. The representative DNA content histograms were shown in the right panel. (c) Overexpression of CDKN2D induced a partial granulocytic differentiation of NB4 cells. Cell surface expression of CD11b in GFP-positive cells was determined by flow cytometry. (d) Wright's staining of NB4 cells. The left panel depicted the MigR1-infected NB4 cells, and the right panel showed MigR1CDKN2D-infected NB4 cells differentiated toward neutrophils. * indicates $P<0.05$
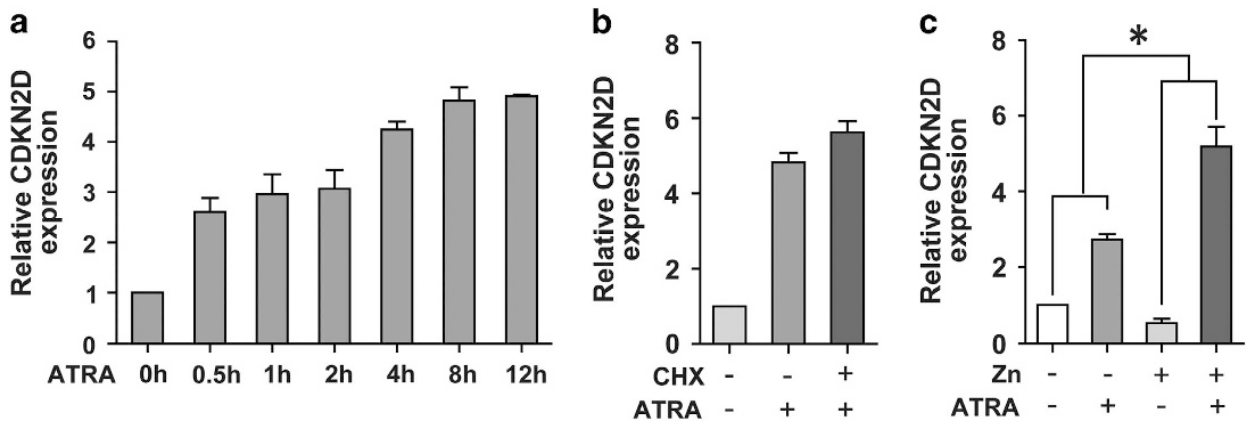

Figure 5 ATRA restores the expression of CDKN2D in APL cells. (a) ATRA restored the expression of CDKN2D mRNA in NB4 cells in a time-dependent manner. NB4 cells were treated with $1 \mu \mathrm{M}$ ATRA for the indicated times. (b) CDKN2D is a direct ATRA-responsive gene. NB4 cells were pretreated with or without $10 \mu \mathrm{g} / \mathrm{ml} \mathrm{cycloheximide} \mathrm{(CHX)} \mathrm{for}$ half an hour and then were cultured for $8 \mathrm{~h}$ in the presence of $1 \mu \mathrm{M}$ ATRA. (c) ATRA induced CDKN2D expression more efficiently in PML/RAR $\alpha$ expressing cells. PR9 cells were pretreated with or without $100 \mu \mathrm{M} \mathrm{ZnSO}_{4}$ for $4 \mathrm{~h}$ and then were cultured for another $12 \mathrm{~h}$ in the presence or absence of $1 \mu \mathrm{M} \mathrm{ATRA}$. ${ }^{*}$ indicates $P<0.05$

Knockdown of CDKN2D impaired the ATRA-induced cell cycle arrest and granulocytic differentiation of APL cells. To further elucidate the function of CDKN2D in ATRA-induced differentiation, we used a siRNA approach to knockdown CDKN2D expression in NB4 cells during ATRA treatment. The reduction of CDKN2D expression in cells transfected with CDKN2D-specific siRNA was confirmed by qRT-PCR and western blot analysis (Figure 6a). Because CDKN2D was involved in regulating both cell cycle and granulocytic differentiation (Figures $4 \mathrm{~b}$ and $\mathrm{c}$ ), we next determined the interference effects of CDKN2D knockdown on cell cycle during ATRA treatment. As shown in Figure 6b, knockdown of CDKN2D expression significantly reduced the percentage of ATRA-induced G0/G1 phase cells from $72.0 \%$ to $58.2 \%$ (Figure $6 \mathrm{~b}$ ), suggesting that CDKN2D was required for ATRA-induced cell cycle arrest of APL cells. Furthermore, we detected the differentiation status of cells during ATRA treatment. As illustrated in Figure 6c, knockdown of CDKN2D 

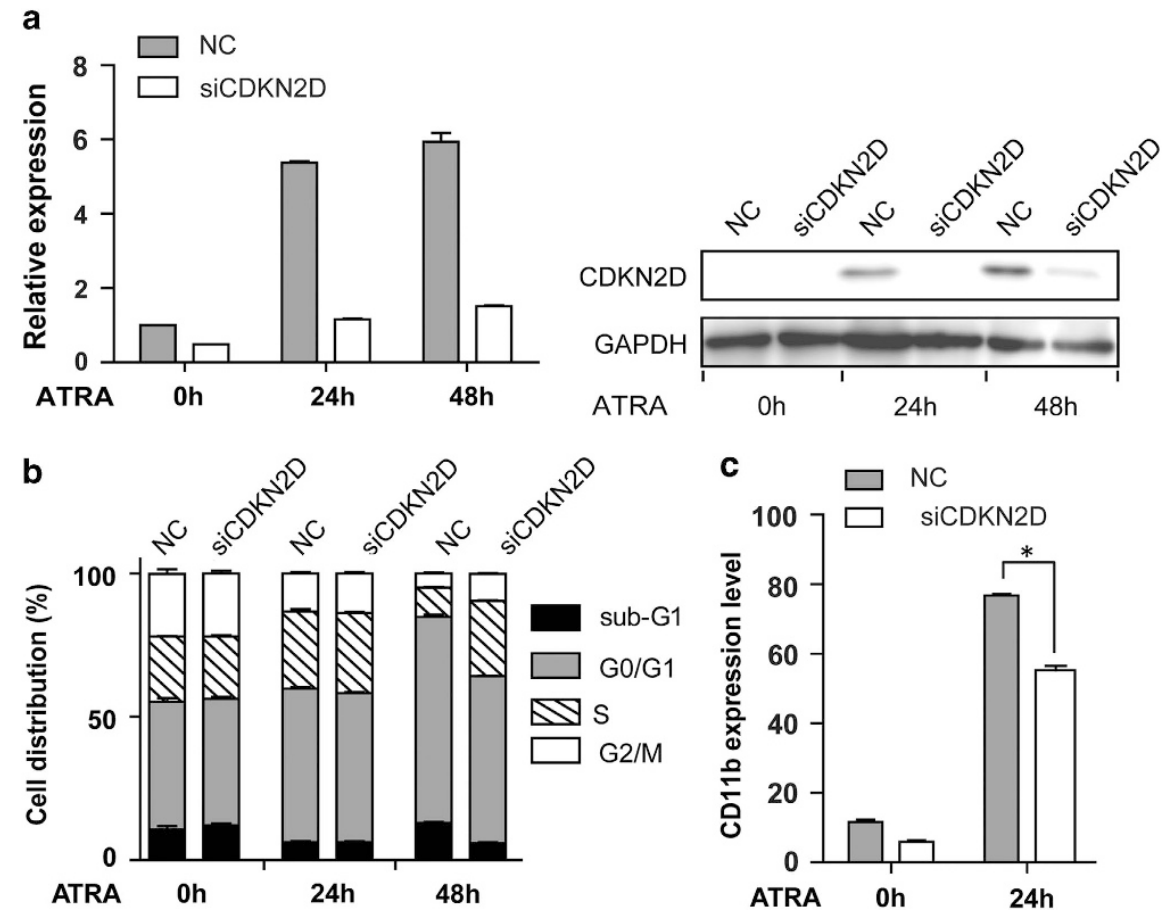

Figure 6 Knockdown of CDKN2D impaired the ATRA-induced cell cycle arrest and granulocytic differentiation of APL cells. (a) The mRNA and protein level of CDKN2D were detected in NB4 cells transfected with siRNA specifically targeting CDKN2D (siCDKN2D) or negative control siRNA (NC). Cells were treated with $1 \mu$ M ATRA for the indicated times. (b) Knockdown of CDKN2D decreased the G0/G1 phase cells induced by ATRA. Error bars showed the S.E.M. from three different assays. (c) Knockdown of CDKN2D impaired granulocytic differentiation of NB4 cells induced by ATRA. Cells were treated with $1 \mu \mathrm{M}$ ATRA for $24 \mathrm{~h} .{ }^{*}$ indicates $P<0.05$

reduced the ATRA-induced differentiation (CD11b) from $76.7 \%$ to $55.2 \%$. We also detected the effect of knockdown of CDKN2D on apoptosis following ATRA treatment and found that CDKN2D had less impact on apoptosis (data not shown). These results support the findings that CDKN2D is required for ATRA-induced cell cycle arrest and differentiation of APL cells.

\section{Discussion}

Leukemia cells are blocked at a certain stage of hematopoietic differentiation and display a high proliferative capacity. In this study, we demonstrated that the reduced expression of $C D K N 2 D$ by the PML/RAR a fusion protein contributed to both the differentiation block and altered proliferation in APL. PML/ RAR $a$ is the initiating factor in the pathogenesis of APL. ${ }^{19}$ Genome-wide studies on the genomic landscape of PML/ RAR $a$ have demonstrated that PML/RAR $a$ targets are involved in a variety of functional processes, including differentiation and proliferation. ${ }^{20}$ However, we know relatively little about the role of PML/RAR $a$ in controlling the coordination of these two processes. Our data provide a mechanism by which PML/RAR $a$ disrupts the coordination between cell proliferation and differentiation by repressing CDKN2D in APL.

Although cell proliferation and differentiation are two mutually exclusive processes, a large body of evidence demonstrates that the two processes are also coordinated and can be controlled by the same regulator. Transcription factors regulating the differentiation process often directly regulate cell cycle progression. For instance, the hematopoietic specific transcription factor GATA1 controls the cell proliferation during erythroid maturation by directly regulating the cyclin-dependent kinase inhibitor p21. ${ }^{21}$ Conversely, key cell cycle regulators also participate in controlling differentiation. One of the most striking examples is cyclin-dependent kinase 6 (CDK6), that is the regulator of cell cycle progression and is capable of blocking myeloid differentiation by inhibiting Runx1 activity. ${ }^{22}$ Our findings provide a new link between cell cycle regulation and granulocytic differentiation through CDKN2D. Therefore, PML/RARa might disrupt both proliferation and differentiation by repressing CDKN2D expression, thus promoting the pathogenesis of APL.

In addition to the well-studied function of CDKN2D on cell cycle regulation, previous studies implicate that CDKN2D might be involved in the differentiation of hematopoietic systems. DMSO induces HL-60 cells to differentiate to granulocyte, accompanied by upregulation of CDKN2C and CDKN2D. ${ }^{23}$ Increased expression of CDKN2D is also involved in the cytokine IL-6-induced growth arrest and the differentiation of M1 leukemic cells. ${ }^{24}$ Overexpressed CDKN2D can induce the differentiation of immature 32Dcl3 myeloid cells to macrophage-like cells in the presence of G-CSF. ${ }^{25}$ Of note, the effects of CDKN2D on differentiation seem to be dependent on the cellular contexts. CDKN2D knockdown shows no effect on differentiation of monocytic leukemia cells U937 induced by 1,25 -VD3 or ATRA. ${ }^{26}$ Here, we showed that the repressed expression of CDKN2D contributed to the differentiation block of APL cells, along with the abnormal proliferation. Furthermore, CDKN2D expression was increased during ATRAinduced differentiation and cell cycle arrest, and knockdown of CDKN2D expression reduced the effect of ATRA on both processes. It should be mentioned that the effect of 
manipulating CDKN2D expression is relatively moderate, compared with the strong effect of ATRA. One possibility was that the transfection efficiency of siRNA did not reach $100 \%$ in all cases, which might cause an underestimation of the CDKN2D effects on cell pool assays because of the impossibility to exclude untransfected cells. Also, as CDK inhibitors usually function redundantly and cooperatively in regulating biological processes, ${ }^{27,28}$ downregulation of CDKN2D may be compensated by other CDK inhibitors. In addition, this may also be partially explained by the fact that CDKN2D is one of the ATRA-responsive genes, which is required but not the only factor in ATRA-induced differentiation and cell cycle arrest. Inhibition of CDKN2D alone is unable to block the effects of other ATRA-responsive genes (data not shown), as numerous genes associated with proliferation (ID1/ID2 ${ }^{29}$ and UBE2D $3^{30}$ ) and differentiation (SPI1 ${ }^{31}$ and CEPBE ${ }^{32}$ ) are induced during ATRA treatment. Collectively, our data show that CDKN2D plays a role in the cell cycle regulation and differentiation of NB4 cells, and more importantly in the coupling of these two processes.

We showed that PML/RARa reduced CDKN2D expression by directly binding to its promoter and transrepressing its transcription. CDKN2D, along with CDKN2A, CDKN2B and CDKN2C, consists of the INK4 family CKIs. Inactivation of genes of the INK4 family is an important event in the pathogenesis of leukemia. For example, deletions of CDKN2A and CDKN2B frequently occur in hematopoietic malignancies. ${ }^{14}$ Loss of CDKN2A increases the growth rate of myeloid colonies in vitro and confers hematopoietic progenitor cells an increased clonal expansion potential. ${ }^{33}$ In addition to gene deletion, hypermethylation of $\mathrm{CpG}$ islands in the promoter regions of $C D K N 2 A$ and $C D K N 2 B$ has also been found to exist in a large percentage of leukemia patients, resulting in the loss of activity of the INK4 family. ${ }^{14}$ However, several clinical studies have shown that gene deletion and hypermethylation are hardly observed in CDKN2D, unlike in other INK4 family CKIs. ${ }^{34}$ In addition, CDKN2D has been reported to be deregulated by PLZF-RARa that is generated from $\mathrm{t}(11 ; 17) \mathrm{APL}$, and is associated with ATRA resistance. ${ }^{35}$ PLZF-RARa promotes leukemogenesis partially by the downregulation of CDKN2D in primary murine hematopoietic progenitors, ${ }^{36}$ which supports the function of CDKN2D in the pathogenesis of APL. These findings add another layer of evidence on the inactivation of the INK4 family genes by repressing their expression directly by the oncogenic fusion protein.

PML/RARa is known to act as a dominant-negative oncoprotein. As it retains the DNA-binding domain of RARa, PML/RARa is usually believed to compete for DNA-binding sites of RARa, resulting in the dominant silencing of RARa target genes. ${ }^{37}$ RARa forms heterodimers with RXR and RARa/RXR usually binds to the canonical RAREs, which consist of direct repeats (DR) of the half consensus ([A/G]G $[\mathrm{G} / \mathrm{T}] \mathrm{TCA})$ with spacing of 2 or 5 (DR2 or DR5). ${ }^{13}$ However, emerging evidence has shown that the DNA-binding spectrum of PML/RARa is more complex and versatile than that of RARa. Owing to the formation of the multimers of PML/RAR $a$ complexes, several studies have shown that the binding sites of PML/RARa may have a variety of spatial arrangements, including other orientations of the two half sites and wide varieties of spacing between the two half sites. ${ }^{38}$ Dmitrii Kamashev et al. ${ }^{38}$ have reported that, in addition to canonical RAREs (DR2 or DR5), PML/RARa can also bind noncanonical RAREs, among which DR4 (DR of two-half sites separated by 4 nucleotides), IR0 (inverted repeat of two-half sites without spacing) and ER8 (everted repeat of two-half sites separated by 8 nucleotides) show stronger binding activity to PML/RARa. These findings suggest that genes whose promoters contain non-canonical RAREs may also be targeted and inhibited by PML/RARa. In accordance with these previous reports, our results demonstrated that PML/ RAR $a$ inhibited the promoter activity of CDKN2D through a non-canonical ER8 site. The feature of PML/RAR $a$ with a more flexible DNA-binding specificity results in a broader spectrum of gene regulated by PML/RARa, thus giving rise to a major gain of function of this fusion protein in the pathogenesis of APL. In the future, it will be very interesting to determine more PML/RAR $a$ targets that participate in the cross-talk between the proliferation and differentiation programs, and finally define the network link the two programs together in APL.

\section{Materials and methods}

Cell culture. NB4, PR9 and U937 cells were cultured in RPMI 1640 medium (Gibco, Carlsbad, CA, USA) supplemented with $10 \%$ (v/v) fetal bovine serum (Gibco), and HEK 293T cells were cultured in Dulbecco's Modified Eagle's Medium (Gibco) supplemented with $10 \%(\mathrm{~V} / \mathrm{v})$ fetal bovine serum, in a humidified atmosphere with $5 \% \mathrm{CO}_{2}$ at $37^{\circ} \mathrm{C}$. ATRA and $\mathrm{ZnSO}_{4}$ (Sigma, St. Louis, MO, USA) were used at final concentrations of $1 \mu \mathrm{M}$ and $100 \mu \mathrm{M}$, respectively.

Plasmid construction and site-directed mutagenesis. A series of sense oligonucleotides-CAGGAGTGAGACCCTGACTCA (-900), TCGTAGTAA GGGCCAATGAATG (-488), GTTGCCACACTCTGACCAATC (-273) and an antisense oligonucleotide-TAACTCACССТСССТССТCC were used to generate a series of 5 ' truncated DNA fragments by PCR using genomic DNA from NB4 cells. The PCR products were cloned into the PGL3 basic vector (Promega, Madison, WI, USA). Mutations of the ER8 site in the CDKN2D (-488) construct were made using the QuikChange site-directed mutagenesis kit (Stratagene, La Jolla, CA, USA) following the manufacturer's protocol. The primers were as follows: sense oligonucleotide 5'-GACCCTGCCCCCAGCCTTTCTCCCTGCCTCAGTTAAACC AGCC-3', antisense oligonucleotide 5'- GGCTGGTTTAACTGAGGCAGGGAGAAA GGCTGGGGGCAGGGTC-3'.

Cell transfection and luciferase reporter assays. HEK 293T cells were seeded at a density of $0.5 \times 10^{5}$ cells/well in 48 -well plates for $24 \mathrm{~h}$ before transfection. A total of $125 \mathrm{ng}$ of the tested promoter (the $900 \mathrm{bp}$ full-length and the different truncations/mutations of the CDKN2D promoter), $2.5 \mathrm{ng}$ of pRL-SV40 and pSG5-PML/RAR $\alpha$ or pSG5 empty vectors (500 ng or increasing amounts in the dose-dependent assay) were transiently transfected into 293T cells using Lipofectamine 2000 (Invitrogen, Carlsbad, CA, USA). After $48 \mathrm{~h}$ of transfection, cells were harvested and luciferase activity was measured with a luminometer (Promega) using Dual-Luciferase Reporter Assay System reagents (Promega). The renilla luciferase plasmid pRL-SV40 was used as control for transfection efficiency.

For NB4 cells, $2 \mu$ g promoter-luciferase reporters or pGL3 basic empty vector and $100 \mathrm{ng}$ pRL-SV40 were transiently transfected into NB4 cells using the Amaxa Nucleofector II device (Lonza, program X-001) with Nucleofector Kit V (Lonza, Cologne, Germany). Twenty-four hours after transfection, cells were harvested. Luciferase activity was measured with a luminometer using Dual-Luciferase Reporter Assay System reagents. The renilla luciferase plasmid pRL-SV40 was used as control for transfection efficiency.

Quantitative reverse transcription-PCR (qRT-PCR). Total RNA was extracted from cells using Trizol Reagent (Invitrogen) according to the manufacturer's protocol. The amount of $2 \mu \mathrm{g}$ total RNA was reverse transcribed into cDNA using random hexamer primers and SuperScript II reverse transcriptase kit (Invitrogen) according to the manufacturer's instruction. Quantitative real-time 
PCR was performed by using $\mathrm{ABI}$ Prism $7900 \mathrm{HT}$ detection system (Applied Biosystems, Foster City, CA, USA) with Power SYBR Green PCR master mix (Applied Biosystems). Relative expression was calculated as a ratio of CDKN2D to GAPDH. Primer sequences of GAPDH and CDKN2D were as follows: GAPDH (forward: 5'-GAAGGTGAAGGTCGGAGTC-3'; reverse: 5'-GAAGATGGTG ATGGGATTTC-3'); CDKN2D (forward: 5'- TCACACTGCTGTGGTCAGCTTT-3'; reverse: 5'- AGGATGTCCACGAGGTCCTGA-3').

ChIP assay. ChIP was performed according to the Affymetrix protocol as previously described. ${ }^{39}$ Briefly, PR9 cells were incubated with $100 \mu \mathrm{M} \mathrm{ZnSO}_{4}$ for $4 \mathrm{~h} ; 5 \times 10^{7}$ cells were then harvested for ChIP assays. A total of $5 \times 10^{7}$ U937 and NB4 cells were harvested for ChIP assays. Cells were crosslinked with $1 \%$ formaldehyde and lysed. Cross-linked chromatin was sonicated to fragments with an average size of 200-1000 bp and immunoprecipitated with specific antibodies against RAR $\alpha$ (C-20x, Santa Cruz Biotechnology, Santa Cruz, CA, USA), PML (H-238x, Santa Cruz Biotechnology) and rabbit lgG (ab46540, Abcam, Cambridge, UK). The total input as well as immunoprecipitated DNA was analyzed by PCR using following primers: positive primer (region 1) (forward: 5'- TAGT GGAACCTCGGATTGGGT-3'; reverse: 5'- TGATTGGTCAGAGTGTGGCAA-3'), negative primer (region 2) (forward: 5'- TGGTCACAAGTCTCTTTTGCATG-3'; reverse: 5'- CACCTACTTAGGCCTCGAGACAG-3'). Each experiment was performed in triplicate and equivalent results were obtained.

RNA interference (siRNA) experiments. The specific sequence used for CDKN2D silencing was selected from the WI siRNA Selection Program (http://sirna.wi.mit.edu). A negative control sequence was used (AGC GUG UAG CUA GCA GAG G). A total of $2 \times 10^{6} \mathrm{NB} 4$ cells were suspended in $100 \mu$ l Amaxa Nucleofector Solution $V$ and mixed with $3 \mu \mathrm{g}$ siRNA. Cells were then transfected by electroporation using Amaxa Nucleofector II device and program $\mathrm{X}$-001. Six hours after transfection, cells were cultured for another $48 \mathrm{~h}$ in the absence or presence of $1 \mu \mathrm{M}$ ATRA. The siRNA sequence targeting CDKN2D was as follows: forward: 5'- GUACCAGUCCAGUCCAUGAUU-3'; reverse: 5'- UCAUGGACUGGACUGGUACUU-3'.

Retroviral constructs. The cDNA encoding full-length human CDKN2D was amplified by RT-PCR from cDNA of NB4 cells and then directionally cloned into an MSCV2.2-derived vector, MigR1, by Xho I and EcoR I sites to form plasmid MigR1-CDKN2D.

Preparation of retroviruses and transfection. The recombinant vectors were transfected into HEK 293T packaging cells using HilyMax (Dojindo, Gaithersburg, MD, USA). The virus-containing cell culture supernatants were collected at 48 and $72 \mathrm{~h}$ after transfection, centrifuged at 1500 r.p.m. for $5 \mathrm{~min}$, aliquoted and stored at $-80^{\circ} \mathrm{C}$. The virus was titred against 3T3-NIH cells.

NB4 cells were seeded at a density of $1 \times 10^{5}$ per well in a six-well culture dish, and infected using viral supernatant and $8 \mu \mathrm{g} / \mathrm{ml}$ Polybrene (Sigma), and centrifuged at $1500 \times g$ for $90 \mathrm{~min}$ at $28^{\circ} \mathrm{C}$. The medium was changed after $6 \mathrm{~h}$, and the cells were cultured normally.

Western blot. Total protein extracts were prepared and western blot analysis was performed as previously described. ${ }^{40}$

Granulocytic differentiation. Granulocytic differentiation of cells was determined by detecting the CD11b expression level. Harvested cells were incubated with anti-CD11b antibody (BD Biosciences, San Jose, CA, USA), and analyzed by a Cytomics FC-500 flow cytometer (Beckman Coulter, Miami, FL, USA). For retroviral transfected cells, GFP-positive cells were analyzed for CD11b expression level.

Cell cycle assay. siRNA-transfected cells were collected, washed twice with PBS and fixed in ice-cold $70 \%$ ethanol overnight at $-20^{\circ} \mathrm{C}$. Then, cells were treated with $100 \mu \mathrm{g} / \mathrm{ml}$ RNase A in PBS, and incubated at $37^{\circ} \mathrm{C}$ for $30 \mathrm{~min}$ before staining with $50 \mu \mathrm{g} / \mathrm{ml}$ of PI (BD Biosciences) for $30 \mathrm{~min}$ at room temperature. Cells were analyzed for DNA content using a Cytomics FC-500 flow cytometer (Beckman Coulter).

For retroviral transfected cells, cells were first fixed with $1 \%$ formaldehyde for $1 \mathrm{~h}$ at $4^{\circ} \mathrm{C}$, washed once with PBS and permeabilized with ice-cold $70 \%$ ethanol overnight at $-20^{\circ} \mathrm{C}$. Then, cells were treated with $100 \mu \mathrm{g} / \mathrm{ml}$ RNase A in PBS, and incubated at $37^{\circ} \mathrm{C}$ for $30 \mathrm{~min}$ before staining with $50 \mu \mathrm{g} / \mathrm{ml}$ of PI for $30 \mathrm{~min}$ at room temperature.
GFP-positive cells were analyzed for DNA content using a Cytomics FC-500 flow cytometer (Beckman Coulter).

Apoptosis analysis. Annexin V-FITC and PI (BD Biosciences) were used to detect apoptotic cells.

Cell morphology. Morphology was determined with Wright's stain of cells centrifuged onto slides by cytospin at 800 r.p.m. for 5 min (Shandon, Runcorn, UK).

Statistical analysis. Statistical significance was determined by a Student's $t$ test. Values were the mean \pm S.E.M. obtained from at least three independent experiments.

\section{Conflict of Interest}

The authors declare no conflict of interest.

Acknowledgements. This work was supported in part by Ministry of Science and Technology Grants of China (2012AA02A211) and National Natural Science Foundation Grants of China (81270625 and 31171257).

1. Ablain J, de The H. Revisiting the differentiation paradigm in acute promyelocytic leukemia Blood 2011; 117: 5795-5802.

2. Wang $Z Y, C$ Chen $Z$. Acute promyelocytic leukemia: from highly fatal to highly curable. Blood 2008; 111: 2505-2515

3. Zhu L, Skoultchi Al. Coordinating cell proliferation and differentiation. Curr Opin Genet Dev 2001; 11: 91-97.

4. Buttitta LA, Katzaroff AJ, Perez CL, de la Cruz A, Edgar BA. A double-assurance mechanism controls cell cycle exit upon terminal differentiation in Drosophila. Dev Cell 2007; 12 : 631-643.

5. Evan Gl, Vousden KH. Proliferation, cell cycle and apoptosis in cancer. Nature 2001; 411: 342-348.

6. Tenen DG. Disruption of differentiation in human cancer: AML shows the way. Nat Rev Cancer 2003; 3: 89-101.

7. Drumea K, Yang ZF, Rosmarin A. Retinoic acid signaling in myelopoiesis. Curr Opin Hematol 2008; 15: 37-41.

8. Wang K, Wang P, Shi J, Zhu X, He M, Jia X et al. PML/RARalpha targets promoter regions containing PU.1 consensus and RARE half sites in acute promyelocytic leukemia. Cancer Cell 2010; 17: 186-197.

9. Rego EM, Wang ZG, Peruzzi D, He LZ, Cordon-Cardo C, Pandolfi PP. Role of promyelocytic leukemia (PML) protein in tumor suppression. J Exp Med 2001; 193 521-529.

10. Salomoni P, Pandolfi PP. The role of PML in tumor suppression. Cell 2002; 108: 165-170.

11. Taghon T, Stolz F, De Smedt M, Cnockaert M, Verhasselt B, Plum J et al. HOX-A10 regulates hematopoietic lineage commitment: evidence for a monocyte-specific transcription factor. Blood 2002; 99: 1197-1204.

12. Buske C, Feuring-Buske M, Antonchuk J, Rosten P, Hogge DE, Eaves CJ et al Overexpression of HOXA10 perturbs human lymphomyelopoiesis in vitro and in vivo. Blood 2001; 97: 2286-2292.

13. Martens JH, Brinkman AB, Simmer F, Francoijs KJ, Nebbioso A, Ferrara F et al. PMLRARalpha/RXR Alters the Epigenetic Landscape in Acute Promyelocytic Leukemia. Cancer Cell 2010; 17: 173-185.

14. Kim WY, Sharpless NE. The regulation of INK4/ARF in cancer and aging. Cell 2006; 127 265-275.

15. Coskun V, Luskin MB. The expression pattern of the cell cycle inhibitor p19(INK4d) by progenitor cells of the rat embryonic telencephalon and neonatal anterio subventricular zone. J Neurosci 2001; 21: 3092-3103.

16. Gilles L, Guieze R, Bluteau D, Cordette-Lagarde V, Lacout C, Favier R et al. P19INK4D links endomitotic arrest and megakaryocyte maturation and is regulated by AML-1. Blood 2008 ; 111: 4081-4091.

17. Tavera-Mendoza L, Wang TT, Lallemant B, Zhang $R$, Nagai $Y$, Bourdeau $V$ et al Convergence of vitamin $\mathrm{D}$ and retinoic acid signalling at a common hormone response element. EMBO Rep 2006; 7: 180-185.

18. Payton JE, Grieselhuber NR, Chang LW, Murakami M, Geiss GK, Link DC et al. High throughput digital quantification of mRNA abundance in primary human acute myeloid leukemia samples. J Clin Invest 2009; 119: 1714-1726.

19. Melnick A, Licht JD. Deconstructing a disease: RARalpha, its fusion partners, and their roles in the pathogenesis of acute promyelocytic leukemia. Blood 1999; 93: 3167-3215.

20. Zheng PZ, Wang KK, Zhang QY, Huang QH, Du YZ, Zhang QH et al. Systems analysis of transcriptome and proteome in retinoic acid/arsenic trioxide-induced cell differentiation/ apoptosis of promyelocytic leukemia. Proc Natl Acad Sci USA 2005; 102: 7653-7658.

21. Papetti M, Wontakal SN, Stopka T, Skoultchi Al. GATA-1 directly regulates p21 gene expression during erythroid differentiation. Cell Cycle 2010; 9: 1972-1980. 
22. Fujimoto T, Anderson K, Jacobsen SE, Nishikawa SI, Nerlov C. Cdk6 blocks myeloid differentiation by interfering with Runx1 DNA binding and Runx1-C/EBPalpha interaction. EMBO J 2007; 26: 2361-2370.

23. Schwaller J, Pabst T, Bickel M, Borisch B, Fey MF, Tobler A. Comparative detection and quantitation of human CDK inhibitor mRNA expression of p15INK4B, p16INK4A, p16beta p18INK4C, p19INK4D, p21WAF1, p27KIP1 and p57KIP2 by RT-PCR using a polycompetitive internal standard. Br J Haematol 1997; 99: 896-900.

24. Narimatsu M, Nakajima K, Ichiba M, Hirano T. Association of Stat3-dependent transcriptional activation of p19INK4D with IL-6-induced growth arrest. Biochem Biophys Res Commun 1997; 238: 764-748.

25. Adachi M, Roussel MF, Havenith K, Sherr CJ. Features of macrophage differentiation induced by p19INK4d, a specific inhibitor of cyclin D-dependent kinases. Blood 1997; 90: 126-137.

26. Tavera-Mendoza LE, Wang TT, White JH. p19INK4D and cell death. Cell Cycle 2006; 5 596-598

27. Thullberg M, Bartkova J, Khan S, Hansen K, Ronnstrand L, Lukas J et al. Distinct versus redundant properties among members of the INK4 family of cyclin-dependent kinase inhibitors. FEBS Lett 2000; 470: 161-166.

28. Ramsey MR, Krishnamurthy J, Pei XH, Torrice C, Lin W, Carrasco DR et al. Expression of p16Ink4a compensates for p18Ink4c loss in cyclin-dependent kinase 4/6-dependent tumors and tissues. Cancer Res 2007; 67: 4732-4741.

29. Nigten J, Breems-de Ridder MC, Erpelinck-Verschueren CA, Nikoloski G, van der Reijden BA van Wageningen $S$ et al. ID1 and ID2 are retinoic acid responsive genes and induce a G0/G1 accumulation in acute promyelocytic leukemia cells. Leukemia 2005; 19: 799-805.

30. Hattori $\mathrm{H}$, Zhang X, Jia Y, Subramanian KK, Jo H, Loison $\mathrm{F}$ et al. RNAi screen identifies UBE2D3 as a mediator of all-trans retinoic acid-induced cell growth arrest in human acute promyelocytic NB4 cells. Blood 2007; 110: 640-650.

31. Mueller BU, Pabst T, Fos J, Petkovic V, Fey MF, Asou $\mathrm{N}$ et al. ATRA resolves the differentiation block in $\mathrm{t}(15 ; 17)$ acute myeloid leukemia by restoring PU.1 expression. Blood 2006; 107: 3330-3338.

32. Park DJ, Chumakov AM, Vuong PT, Chih DY, Gombart AFMiller WH Jr. et al. CCAAT/enhancer binding protein epsilon is a potential retinoid target gene in acute promyelocytic leukemia treatment. J Clin Invest 1999; 103: 1399-1408.

33. Lewis JL, Chinswangwatanakul W, Zheng B, Marley SB, Nguyen DX, Cross NC et al. The influence of INK4 proteins on growth and self-renewal kinetics of hematopoietic progenitor cells. Blood 2001; 97: 2604-2610.
34. Shiohara M, Spirin K, Said JW, Gombart AF, Nakamaki T, Takeuchi S et al. Alterations of the cyclin-dependent kinase inhibitor p19 (INK4D) is rare in hematopoietic malignancies. Leukemia 1996; 10: 1897-1900.

35. Licht JD, Chomienne C, Goy A, Chen A, Scott AA, Head DR et al. Clinical and molecular characterization of a rare syndrome of acute promyelocytic leukemia associated with translocation (11:17). Blood 1995; 85: 1083-1094.

36. Rice KL, Hormaeche I, Doulatov S, Flatow JM, Grimwade D, Mills KI et al. Comprehensive genomic screens identify a role for PLZF-RARalpha as a positive regulator of cell proliferation via direct regulation of c-MYC. Blood 2009; 114: 5499-5511.

37. de Thé $H$, Chen $Z$. Acute promyelocytic leukaemia: novel insights into the mechanisms of cure. Nat Rev Cancer 2010; 10: 775-783.

38. Kamashev D, Vitoux D, De The H. PML-RARA-RXR oligomers mediate retinoid and rexinoid/ cAMP cross-talk in acute promyelocytic leukemia cell differentiation. J Exp Med 2004; 199: 1163-1174.

39. Carroll JS, Liu XS, Brodsky AS, Li W, Meyer CA, Szary AJ et al. Chromosome-wide mapping of estrogen receptor binding reveals long-range regulation requiring the forkhead protein FoxA1. Cell 2005; 122: 33-43.

40. Jiang $\mathrm{Y}$, Xue ZH, Shen WZ, Du KM, Yan H, Yu Y et al. Desferrioxamine induces leukemic cell differentiation potentially by hypoxia-inducible factor-1 alpha that augments transcriptional activity of CCAAT/enhancer-binding protein-alpha. Leukemia 2005; 19: $1239-1247$.

(c) (1) () $\odot$ Cell Death and Disease is an open-access journal cublished by Nature Publishing Group. This work is licensed under a Creative Commons Attribution-NonCommercialNoDerivs 3.0 Unported License. The images or other third party material in this article are included in the article's Creative Commons license, unless indicated otherwise in the credit line; if the material is not included under the Creative Commons license, users will need to obtain permission from the license holder to reproduce the material. To view a copy of this license, visit http://creativecommons. org/licenses/by-nc-nd/3.0/ 